\title{
MULTIPARAMETER MAXIMAL FUNCTIONS ALONG DILATION-INVARIANT HYPERSURFACES
}

$\mathrm{BY}$

\author{
HASSE CARLSSON, PETER SJÖGREN AND JAN-OLOV STRÖMBERG
}

\begin{abstract}
Consider the hypersurface $x_{n+1}=\prod_{1}^{n} x_{i}^{\alpha_{i}}$ in $\mathbf{R}^{n+1}$. The associated maximal function operator is defined as the supremum of means taken over those parts of the surface lying above the rectangles $\left\{0 \leq x_{i} \leq h_{i}, i=\right.$ $1, \ldots, n\}$. We prove that this operator is bounded on $L^{p}$ for $p>1$. An analogous result is proved for a quadratic surface in $\mathbf{R}^{3}$.
\end{abstract}

1. Introduction. Let $K$ be the right circular cone $z^{2}=x^{2}+y^{2}, z>0$, in $\mathbf{R}^{3}$. We fix a generating ray, say $l_{0}=\{(t, 0, t): t>0\}$, and consider portions of $K$ bounded by $l_{0}$, a variable generating ray, and a sphere centered at the origin. Thus, we set

$$
A_{r \theta}=\left\{(x, y, z) \in K: 0<\arg (x+i y)<\theta, x^{2}+y^{2}+z^{2}<r^{2}\right\} .
$$

The area measure on $K$ is $d S=\sqrt{2} d x d y$, and we study mean values

$$
m_{r \theta} f(x, y, z)=\left(\int_{A_{r \theta}} d S\right)^{-1} \int_{A_{r \theta}} f\left(x-x^{\prime}, y-y^{\prime}, z-z^{\prime}\right) d S\left(x^{\prime}, y^{\prime}, z^{\prime}\right) .
$$

We ask whether $m_{r \theta} f \rightarrow f$ a.e. as $r \rightarrow 0$ and $\theta$ varies freely in $(0,2 \pi]$ when $f \in L^{p}$.

It is well known that to prove such a.e. convergence, one must show that the corresponding maximal function operator

$$
M_{K} f(x, y, z)=\sup _{r, \theta} m_{r \theta}|f|(x, y, z)
$$

is bounded on $L^{p}$ or, at least, of weak type $(p, p)$. When $\theta=2 \pi$, it is easy to control $m_{r \theta}|f|$ via the mean of the one-dimensional maximal functions of $|f|$ along all the generating rays. This mean can also be used when $\theta$ is large, say $\theta \geq \pi / 4$. The corresponding part of $M_{K}$ is therefore bounded on $L^{p}, p>1$. To handle small $\theta$, notice that $A_{r \theta}$ is the disjoint union of sets

$$
A_{r \gamma}^{\prime}=\left\{(x, y, z) \in K: x, y>0, \gamma / 2<y / x<\gamma, r^{2} / 4<x^{2}+y^{2}+z^{2}<r^{2}\right\} .
$$

Therefore, we can use $A_{r \gamma}^{\prime}$ instead of $A_{r \theta}$ when defining $M_{K}$.

If we change coordinates by letting

$$
x_{1}=\frac{z+x}{\sqrt{2}}, \quad x_{2}=\frac{z-x}{\sqrt{2}}, \quad x_{3}=y,
$$

the equation of $K$ becomes $x_{3}= \pm \sqrt{2 x_{1} x_{2}}, x_{1}, x_{2} \geq 0$, and we take only the positive sign here. Now $A_{r \gamma}^{\prime}$ corresponds to

$$
A_{r \beta}^{\prime \prime}=\left\{\left(x_{1}, x_{2}, \sqrt{2 x_{1} x_{2}}\right): \beta^{\prime}<x_{2} / x_{1}<\beta, r / 2<x_{1}+x_{2}<r\right\},
$$

Received by the editors June 27, 1984.

1980 Mathematics Subject Classification. Primary 42B25. 
where $\beta=\left(\sqrt{\gamma^{2}+1}-1\right) /\left(\sqrt{\gamma^{2}+1}+1\right)$ is roughly $\gamma^{2} / 4$, and $\beta^{\prime} \approx \beta / 4$. In each such set, $d S / d x_{1} d x_{2}=\left(1+\frac{1}{2}\left(x_{1} / x_{2}+x_{2} / x_{1}\right)\right)^{1 / 2}$ is approximately constant. Therefore, when forming means, we can use $d x_{1} d x_{2}$ instead of $d S$.

We next replace the sets $A_{r \beta}^{\prime \prime}$ by subsets of $K$ whose projections on the $x_{1} x_{2}$ plane are rectangles; these are more convenient to work with. Since $\theta, \gamma$, and $\beta$ are small, $x_{1}+x_{2}$ is roughly $x_{1}$ in $A_{r \beta}^{\prime \prime}$, and $x_{1}$ and $x_{2}$ are approximately constant there. Thus, we can cover $A_{r \beta}^{\prime \prime}$ by a small number of disjoint sets of type

$$
\left\{\left(x_{1}, x_{2}, \sqrt{2 x_{1} x_{2}}\right): h_{1} / 2<x_{1}<h_{1}, h_{2} / 2<x_{2}<h_{2}\right\}, \quad h_{1}, h_{2}>0,
$$

whose total measure is not much larger than that of $A_{r \beta}^{\prime \prime}$. Therefore, $M_{K} f$ is dominated by the supremum of means over sets of this type or, equivalently, by $M f$, where

$M f\left(y_{1}, y_{2}, y_{3}\right)=\sup _{h_{1}, h_{2}>0} \frac{1}{h_{1} h_{2}} \int_{0}^{h_{1}} d x_{1} \int_{0}^{h_{2}} d x_{2}\left|f\left(y_{1}-x_{1}, y_{2}-x_{2}, y_{3}-\sqrt{2 x_{1} x_{2}}\right)\right|$.

This leads us to a more general problem in $\mathbf{R}^{n+1}=\left\{x=\left(x^{\prime}, x_{n+1}\right) \in \mathbf{R}^{n} \times \mathbf{R}\right\}$. Let $x_{n+1}=F\left(x^{\prime}\right)$ be the equation of a hypersurface, defined at least for $x_{1}, \ldots, x_{n}>$ 0 . Define

(1.1) $M f(y)=\sup _{h_{1}, \ldots, h_{n}>0} \frac{1}{h_{1} \cdots h_{n}} \int_{0}^{h_{1}} d x_{1} \cdots \int_{0}^{h_{n}} d x_{n}\left|f\left(y^{\prime}-x^{\prime}, y_{n+1}-F\left(x^{\prime}\right)\right)\right|$.

In $\left[8\right.$, Problem 8, p. 1289], Stein and Wainger raised the question of $L^{p}$ boundedness for such maximal operators. We shall deal mainly with the case

$$
x_{n+1}=F\left(x^{\prime}\right)=\prod_{i=1}^{n} x_{i}^{\alpha_{i}}, \quad \alpha_{i} \in \mathbf{R}, i=1, \ldots, n .
$$

THEOREM 1. If $F$ is as in (1.2), $M$ is well defined and bounded on $L^{p}\left(\mathbf{R}^{n+1}\right)$ for all $p>1$.

This theorem applies to the maximal function associated with the surface $x_{1} x_{2}=$ $x_{3} x_{4}$ in $\mathbf{R}^{4}$, which appears in connection with admissible convergence in the symmetric space $\mathrm{SL}(4, \mathbf{R}) / \mathrm{SO}(4, \mathbf{R})$. Sjögren $[\mathbf{6}]$ proves admissible convergence in a general symmetric space.

By standard techniques, Theorem 1 implies a convergence result. Assume $\alpha_{i} \geq 0$ for all $i$ and $\alpha_{i}>0$ for some $i$. If $f \in L^{p}\left(\mathbf{R}^{n+1}\right)$, the mean

$$
\frac{1}{h_{1} \cdots h_{n}} \int_{0}^{h_{1}} d x_{1} \cdots \int_{0}^{h_{n}} d x_{n} f\left(y^{\prime}-x^{\prime}, y_{n+1}-F\left(x^{\prime}\right)\right)
$$

tends to $f(y)$ as $h_{1}, \ldots, h_{n} \rightarrow 0$, for almost all $y \in \mathbf{R}^{n+1}$.

The Hilbert transform associated with the hypersurface (1.2) and similar surfaces of lower dimension has been studied by Nagel and Wainger $[\mathbf{4}]$ and by Vance $[\mathbf{1 1}]$ and Strichartz $[\mathbf{1 0}]$.

Theorem 1 is proved in $\S 2$. The method is an adaptation of the known methods for maximal functions along curves, involving Fourier transforms and analytic interpolation as in [8]. Theorem 1 implies that the operator $M_{K}$ defined above is bounded on $L^{p}, p>1$. However, this boundedness can be proved in a simpler way by means of one-dimensional maximal functions in lacunar directions, as shown in 
$\S 3$. When $n=2$, this proof also applies to those surfaces of type (1.2) which are conical, i.e., for which $\alpha_{1}+\alpha_{2}=1$. $\S 3$ also contains a proof of the following result for a second-degree surface in $\mathbf{R}^{3}$.

THEOREM 2. Let $n=2$ and $F\left(x_{1}, x_{2}\right)=a x_{1}^{2}+b x_{1} x_{2}+c x_{2}^{2}$, where $a, b, c \in \mathbf{R}$. Then $M$ is bounded on $L^{p}$ for $p>1$.

The proof uses combinations of maximal functions along curves and Theorem 1 .

2. Proof of Theorem 1. We first eliminate those $\alpha_{i}$ which are zero. If $\alpha_{i}=0$, then $F$ is independent of $x_{i}$. In the mean value in (1.1), we can then estimate the integral

$$
\frac{1}{h_{i}} \int_{0}^{h_{i}}\left|f\left(y^{\prime}-x^{\prime}, y_{n+1}-F\left(x^{\prime}\right)\right)\right| d x_{i}
$$

by means of the one-dimensional maximal function taken in the $x_{i}$ direction. The remaining integral defines, for each fixed $y_{i}$, a maximal function of the same type in a lower-dimensional space. Continuing in this way, we can reduce the problem to the case where no $\alpha_{i}$ vanishes. We also assume that we are not in the case $n=1, \alpha_{1}=1$, which is trivial.

We need some notation. As usual, $C$ denotes various constants. Let

$$
J=\left\{j=\left(j_{1}, \ldots, j_{n+1}\right) \in \mathbf{Z}^{n+1}: j_{n+1}=\sum_{i=1}^{n} j_{i} \operatorname{sgn} \alpha_{i}\right\} .
$$

Define $a=\left(a_{1}, \ldots, a_{n+1}\right)$ by the conditions $a_{1}^{\left|\alpha_{1}\right|}=\cdots=a_{n}^{\left|\alpha_{n}\right|}=a_{n+1}=2$. The surface (1.2) is invariant under the dilations $\delta_{j} x=\left(a_{1}^{j_{1}} x_{1}, \ldots, a_{n+1}^{j_{n+1}} x_{n+1}\right)$, provided $j \in J$. The Jacobian of $\delta_{j}$ is $a^{j}=\prod_{i=1}^{n+1} a_{i}^{j_{i}}$. If $j^{\prime} \in \mathbf{Z}^{n}$ and $x^{\prime}=\left(x_{1}, \ldots, x_{n}\right) \in \mathbf{R}^{n}$, we set $\delta_{j^{\prime}} x^{\prime}=\left(a_{1}^{j_{1}} x_{1}, \ldots, a_{n}^{j_{n}} x_{n}\right)$. The dilations $\lambda_{j}$ of a measure or a distribution $\lambda$ in $\mathbf{R}^{n+1}$ are defined by

$$
\int \varphi d \lambda_{j}=\int \varphi\left(\delta_{j} x\right) d \lambda
$$

for test functions $\varphi$. The maximal function operator associated with $\lambda$ is $M_{\lambda} \varphi=$ $\sup _{j \in J}\left|\lambda_{j} * \varphi\right|$.

We shall dominate $M f$ by means of $M_{\mu} f$, where $\mu$ will be a compactly supported smooth measure on the surface. Let $\psi \in C_{0}^{\infty}\left(\mathbf{R}^{n}\right)$ be a nonnegative function with support in $\left\{x^{\prime} \in \mathbf{R}^{n}: x_{i}>0, i=1, \ldots, n\right\}$ which equals 1 in $R=\left\{x^{\prime} \in \mathbf{R}^{n}: 1 \leq\right.$ $\left.x_{i} \leq a_{i}\right\}$. Now $\mu$ is defined by

$$
\int \varphi d \mu=\int \varphi\left(x^{\prime}, F\left(x^{\prime}\right)\right) \psi\left(x^{\prime}\right) d x^{\prime}, \quad \varphi \in C\left(\mathbf{R}^{n+1}\right) .
$$

Let $f \geq 0$ be measurable in $\mathbf{R}^{n+1}$. Then $f\left(y^{\prime}-x^{\prime}, y_{n+1}-F\left(x^{\prime}\right)\right)$ is measurable in $x^{\prime}$ for almost every $y$. In (1.1) it is enough to consider $h_{i}$ which are of the form $a_{i}^{m_{i}}$, with integers $m_{1}, \ldots, m_{n}$. Then the domain of integration $\left\{x^{\prime} \in \mathbf{R}^{n}: 0 \leq\right.$ $\left.x_{i} \leq h_{i}\right\}$ is the union of those sets $\delta_{-j^{\prime}} R$ for which $j^{\prime}=\left(j_{1}, \ldots, j_{n}\right) \in \mathbf{Z}^{n}$ satisfies $j_{i}>-m_{i}, i=1, \ldots, n$. Thus, the mean in (1.1) is bounded by the supremum of the means over $\delta_{-j^{\prime}} R$. But these last means are dominated by $\mu_{j} * f$ with $j \in J$ and, thus, by $M_{\mu} f$. Hence, $M f \leq C M_{\mu} f$, and the following result implies Theorem 1 . 
PROPOSITION 1. If $f \in L^{p}\left(\mathbf{R}^{n+1}\right), p>1$, the convolution $\mu_{j} * f$ is well defined almost everywhere for $j \in J$, and $\left\|M_{\mu} f\right\|_{p} \leq C\|f\|_{p}$.

The $L^{2}$-estimate here can be proved by means of a $g$-function argument. But for $1<p<2$, we need to consider an analytic family of operators. Let $\mu^{z}=G_{z} * \mu, z \in$ C, where the distribution $G_{z}$ is defined by $\hat{G}_{z}(\xi)=\left(1+|\xi|^{2}\right)^{-z / 2}$. The following two lemmas will allow analytic interpolation.

Lemma 1. There exists a $\sigma>0$ such that when $-\sigma<\operatorname{Re} z<0$,

$$
\left\|M_{\mu^{z}} f\right\|_{2} \leq C(z)\|f\|_{2}, \quad f \in S .
$$

Lemma 2. For $0<\operatorname{Re} z<1$ and each $p>1$,

$$
\left\|M_{\mu^{z}} f\right\|_{p} \leq C(z)\|f\|_{p}, \quad f \in S .
$$

Here and in the sequel, $C(z)$ denotes constants which are bounded, for fixed $\operatorname{Re} z$, by a polynomial in $|z|$.

PROOF OF LEMMA 1. We shall compare $\mu$ to a smooth measure $\nu$ such that the Fourier transform $\hat{\mu}-\hat{\nu}$ is small near the coordinate hyperplanes. This will allow us to estimate a $g$-function. Let $0 \leq \phi \in C_{0}^{\infty}(-1,1)$ with $\int \phi d x=1$, and let $\delta$ denote the Dirac measure in $\mathbf{R}$. Then we set

$$
\nu=\mu-\mu * \bigotimes_{i=1}^{n+1}(\delta-\phi) .
$$

The support of $\nu$ is compact, and we claim that $\nu$ is a $C^{\infty}$ function. Clearly $\nu$ is a sum of terms obtained by convolving $\mu$ with $\phi$ in one or more variables. If we convolve $\mu$ with $\phi$ in the $(n+1)$ st variable, we get the $C^{\infty}$ function $\psi\left(x^{\prime}\right) \phi\left(x_{n+1}-F\left(x^{\prime}\right)\right)$. Convolution with $\phi$ in other variables preserves $C^{\infty}$, so those terms where $\mu$ is convolved with $\phi$ in the $(n+1)$ st variable are $C^{\infty}$. Next, consider a term where $\mu$ is convolved with $\phi$ in the $i$ th variable, $i \neq n+1$. Then we solve the equation $x_{n+1}=F\left(x^{\prime}\right)$ for $x_{i}$ and replace $x_{n+1}$ by $x_{i}$ in the argument just given. Notice that this change of variables introduces a Jacobian in the expression for $\mu$ which is $C^{\infty}$ in $\operatorname{supp} \mu$. Hence, $\nu \in C_{0}^{\infty}$.

We estimate $\hat{\mu}-\hat{\nu}$ next. Since $\hat{\mu}$ is bounded and $\hat{\phi}(0)=1$, one has

$$
|\hat{\mu}(\xi)-\hat{\nu}(\xi)| \leq C\left|\xi_{i}\right|, \quad i=1, \ldots, n .
$$

To estimate $\hat{\mu}$, and thus $\hat{\mu}-\hat{\nu}$, at infinity, we use a version of van der Corput's lemma (see Littman [1] ). The determinant of the Hessian $\left(\partial^{2} F / \partial x_{i} \partial x_{j}\right)_{i, j=1}^{n}$ is

$$
(-1)^{n}\left(1-\sum_{i=1}^{n} \alpha_{i}\right) F\left(x^{\prime}\right) \prod_{i=1}^{n} \frac{\alpha_{i}}{x_{i}^{2}} .
$$

This expression vanishes only when $\sum_{i=1}^{n} \alpha_{i}=1$, and then $\sum_{i=1}^{n-1} \alpha_{i} \neq 1$, so that the Hessian has rank at least $n-1$. Therefore, $[\mathbf{1}]$ gives $|\hat{\mu}(\xi)| \leq C|\xi|^{-\gamma}$, where $\gamma \geq \frac{1}{2}(n-1)$ and $\gamma \geq \frac{1}{2}$ since we have excluded the case $n=1, \alpha_{1}=1$. Since $\hat{\nu} \in S$, we may summarize:

$$
|\hat{\mu}(\xi)-\hat{\nu}(\xi)| \leq C \min \left((1+|\xi|)^{-\gamma},\left|\xi_{1}\right|, \ldots,\left|\xi_{n}\right|\right) .
$$


Here we can replace each $\left|\xi_{i}\right|$ by $\left|\xi_{i}\right|^{\varepsilon}$ for $0<\varepsilon<1$. Estimating the minimum by a geometric mean, one has

$$
|\hat{\mu}(\xi)-\hat{\nu}(\xi)| \leq C(1+|\xi|)^{-\gamma / 2} \prod_{i=1}^{n}\left|\xi_{i}\right|^{\varepsilon / 2 n} .
$$

Letting $\nu^{z}=G_{z} * \nu$, we get

$$
\begin{aligned}
\left|\hat{\mu}^{z}(\xi)-\hat{\nu}^{z}(\xi)\right| & \leq C(1+|\xi|)^{-\gamma / 2-\operatorname{Re} z} \prod_{i=1}^{n}\left|\xi_{i}\right|^{\varepsilon / 2 n} \\
& \leq C \prod_{i=1}^{n}\left(1+\left|\xi_{i}\right|\right)^{-(\gamma / 2+\operatorname{Re} z) / n}\left|\xi_{i}\right|^{\varepsilon / 2 n}
\end{aligned}
$$

for $\operatorname{Re} z>-\frac{1}{2} \gamma$. If $\varepsilon$ is small, the assumptions of the following lemma will be satisfied.

LEMMA 3. If $\lambda \in \mathcal{S}^{\prime}\left(\mathbf{R}^{n+1}\right)$ satisfies $|\hat{\lambda}(\xi)| \leq \prod_{i=1}^{n} \min \left(\left|\xi_{i}\right|^{\eta},\left|\xi_{i}\right|^{-\eta}\right)$ for some $\eta>0$, then for $f \in S\left(\mathbf{R}^{n+1}\right)$,

$$
\left\|M_{\lambda} f\right\|_{2} \leq C\|f\|_{2}, \quad C=C(\eta, n) .
$$

PROOF. We have $M_{\lambda} f \leq\left(\sum_{j \in J}\left|\lambda_{j} * f\right|^{2}\right)^{1 / 2}$, and thus, by Plancherel's theorem,

$$
\left\|M_{\lambda} f\right\|_{2} \leq \sup _{\xi}\left(\sum_{j \in J}\left|\hat{\lambda}_{j}(\xi)\right|^{2}\right)^{1 / 2}\|f\|_{2} .
$$

We must show that the supremum appearing here is finite. Since $\hat{\lambda}_{j}(\xi)=\hat{\lambda}\left(\delta_{j} \xi\right)$, we get

$$
\sum_{j \in J}\left|\hat{\lambda}_{j}(\xi)\right|^{2} \leq \prod_{i=1}^{n} \sum_{j_{i} \in \mathbf{Z}} \min \left(\left|a_{i}^{j_{i}} \xi_{i}\right|^{2 \eta},\left|a_{i}^{j_{i}} \xi_{i}\right|^{-2 \eta}\right) \leq C,
$$

which proves the lemma.

By this lemma, $M_{\mu^{z}-\nu^{z}}$ is bounded on $L^{2}\left(\mathbf{R}^{n+1}\right)$, with norm depending only on $\operatorname{Re} z$. To finish the proof of Lemma 1, we must therefore estimate $M_{\nu^{z}}$. By taking derivatives of $\hat{\nu}^{z}$ of order $n+2$, we get

$$
\left|\nu^{z}(x)\right| \leq C(z)(1+|x|)^{-n-2}
$$

because $\hat{\nu} \in S$. Therefore, $M_{\nu^{z}}$ is dominated by the strong maximal function, and Lemma 1 follows.

Proof of Lemma 2. Let $0<\operatorname{Re} z<1$. The behavior of $G_{z}$ is well known; see e.g. [7, Chapter V, §3]. One has

$$
\left|G_{z}(x)\right| \leq C(z) \begin{cases}|x|^{\operatorname{Re} z-n-1}, & |x| \leq 1 \\ |x|^{-N}, & |x|>1\end{cases}
$$

for any $N$, which could also be verified directly. Choosing $N=n+1+\operatorname{Re} z$, we conclude

$$
\left|G_{z}\right| \leq C(z) \sum_{m \in \mathbf{Z}} 2^{-|m| \operatorname{Re} z+m(n+1)} \chi_{|x|<2^{-m}}
$$


where $\chi$ denotes characteristic function. This implies

$$
\begin{aligned}
\left|\mu_{j}^{z} * f(x)\right| & =\left|\iint f\left(x-\delta_{j}(y+u)\right) G_{z}(y) d y d \mu(u)\right| \\
& \leq C(z) \sum_{m \in \mathbf{Z}} 2^{-|m| \operatorname{Re} z} \int d \mu(u) \int_{|y|<2-m} 2^{m(n+1)}\left|f\left(x-\delta_{j} u-\delta_{j} y\right)\right| d y .
\end{aligned}
$$

The inner integral here is at most

$$
2^{m(n+1)} a^{-j} \int_{\left|y_{i}\right|<2^{-m} a_{i}^{j_{i}}}\left|f\left(x-\delta_{j} u-y\right)\right| d y \leq M_{1}\left(u_{1}, m\right) \cdots M_{n+1}\left(u_{n+1}, m\right) f(x) .
$$

By $M_{i}\left(u_{i}, m\right)$ we mean the one-dimensional maximal operator

$$
M_{i}\left(u_{i}, m\right) g(t)=\sup _{k \in \mathbf{Z}} 2^{m} a_{i}^{-k} \int_{|s|<2^{-m} a_{i}^{k}}\left|g\left(t-a_{i}^{k} u_{i}-s\right)\right| d s, \quad g \in L_{\text {loc }}^{1}(\mathbf{R}),
$$

acting in the $i$ th variable. Thus,

$$
\left\|M_{\mu^{z}} f\right\|_{p} \leq C(z) \sum_{m \in \mathbf{Z}} 2^{-|m| \operatorname{Re} z} \int\left\|M_{1}\left(u_{1}, m\right) \cdots M_{n+1}\left(u_{n+1}, m\right) f\right\|_{p} d \mu(u) .
$$

From Lemma 4 it follows that each $M_{i}\left(u_{i}, m\right)$ is bounded on $L^{p}, p>1$, with norm at most $C(\max (1, m))^{1 / p}$, uniformly for $u \in \operatorname{supp} \mu$. This implies

$$
\left\|M_{\mu^{z}} f\right\|_{p} \leq C(z) \sum_{m \in \mathbf{Z}} 2^{-|m| \operatorname{Re} z}(\max (1, m))^{(n+1) / p}\|f\|_{p} \leq C(z)\|f\|_{p}
$$

which ends the proof of Lemma 2.

To state Lemma 4 we take a decreasing sequence $\omega=\left(\omega_{k}\right)_{-\infty}^{+\infty}$ of positive numbers and a sequence $\tau=\left(\tau_{k}\right)_{-\infty}^{+\infty}$ of real numbers. Consider the maximal function

$$
M^{\omega, \tau} g(t)=\sup _{k \in \mathbf{Z}} \frac{1}{2 \omega_{k}} \int_{-\omega_{k}}^{\omega_{k}}\left|g\left(t-\tau_{k}-s\right)\right| d s, \quad g \in L_{\text {loc }}^{1}(\mathbf{R}) .
$$

If the translations $\tau_{k}$ are no larger than the corresponding interval lengths $\omega_{k}$, then $M^{\omega, \tau}$ is essentially the classical maximal function operator. But one can allow $\left|\tau_{k}\right| \gg \omega_{k}$ under a condition which compares $\tau_{k}$ to the quantities $\omega_{k^{\prime}}, k^{\prime} \leq k$.

LEMMA 4. Assume there exists an integer $m \geq 0$ such that for each $k$ the number of $l$ 's with $l \geq k$ and $\left|\tau_{l}\right|>\omega_{k}$ is at most $m$. Then $M^{\omega, \tau}$ is of weak type $(1,1)$ with constant at most $C \cdot(m+1)$, and $M^{\omega, \tau}$ is bounded on $L^{p}, p>1$, with norm at most $C \cdot(m+1)^{1 / p}, C=C(p)$.

This lemma is a consequence of Theorem 4 in Nagel and Stein [2]. It can also be proved by means of Lemma 2 in Sjögren [5]. However, we give a direct proof here.

PRoOF. Fix $g \in L^{1}(\mathbf{R})$ and $\alpha>0$. Let $F_{k}$ be the family of all intervals $I$ of length $2 \omega_{k}$ such that $\int_{I}|g| d s>\alpha|I|$. Then

$$
\left\{M^{\omega, \tau} g>\alpha\right\} \subset \bigcup_{k} \bigcup_{I \in \mathcal{F}_{k}}\left(I+\tau_{k}\right) .
$$


By a standard covering argument, one can find a disjoint subcollection $\left\{I_{n}\right\}_{n}$ of $\mathcal{F}=\bigcup_{k} \mathcal{F}_{k}$ such that each $I \in \mathcal{F}$ is contained in $5 I_{n}$ for some $n$. We shall show that for each $n$,

$$
\left|\bigcup_{k} \bigcup_{\substack{I \in \mathcal{F}_{k} \\ I \subset 5 I_{n}}}\left(I+\tau_{k}\right)\right| \leq C(m+1)\left|I_{n}\right| .
$$

To this end, we fix $n$ and let $k_{0} \in \mathbf{Z} \cup\{ \pm \infty\}$ be defined by

$$
k_{0}=\inf \left\{k: 2 \omega_{k} \leq 5\left|I_{n}\right|\right\} .
$$

We shall divide the range of $k$ in (2.2) into three subsets. Take $k \in \mathbf{Z}$, and let $I \in \mathcal{F}_{k}, I \subset 5 I_{n}$. If $k<k_{0}$, then $I \in \mathcal{F}_{k}$ is too long to be contained in $5 I_{n}$. If $k \geq k_{0}$ and $\left|\tau_{k}\right| \leq 5\left|I_{n}\right| / 2$, then $I+\tau_{k} \subset 10 I_{n}$. In the remaining case, $k \geq k_{0},\left|\tau_{k}\right|>$ $5\left|I_{n}\right| / 2$, one has $\left|\tau_{k}\right|>\omega_{k_{0}}$. (If $k_{0}=-\infty$, we define $\omega_{-\infty}$ as the obvious limit.) By hypothesis, this occurs for at most $m$ values of $k$. For each such $k$, we get $I+\tau_{k} \subset 5 I_{n}+\tau_{k}$.

By summation we obtain (2.2). Now (2.1), (2.2) imply

$$
\left|\left\{M^{\omega, \tau} g>\alpha\right\}\right| \leq C(m+1) \sum\left|I_{n}\right| \leq C(m+1) \alpha^{-1}\|g\|_{1},
$$

and the weak type $(1,1)$ inequality is proved.

The strong $L^{p}$ estimate follows by interpolation, since $M^{\omega, \tau}$ is bounded on $L^{\infty}$. This completes the proof of Lemma 4.

PROOF OF PROPOSITION 1. We shall apply the analytic interpolation theorem (see $[\mathbf{9}$, Chapter V, §4]) to Lemmas 1 and 2 . First we must linearize the operators. Let $N$ be a natural number and $j(x)$ a measurable mapping of $\mathbf{R}^{n+1}$ into $\{j \in$ $\left.J:\left|j_{i}\right| \leq N, i=1, \ldots, n\right\}$. Then $\mu_{j(x)}^{z} * f(x)$ varies linearly with $f \in S$. The interpolation theorem requires simple functions. Therefore, we let $\Phi \in C_{0}^{\infty}$ be nonnegative and satisfy $\int \Phi d x=1$. Consider the mapping

$$
T_{z} g(x)=\mu_{j(x)}^{z} * \Phi * g(x),
$$

which is defined for simple $g$, since then $\Phi * g \in S$. By Lemmas 1 and 2 , the inequality $\left\|T_{z} g\right\|_{p} \leq C(z)\|g\|_{p}$ holds for $p=2$ if $-\sigma<\operatorname{Re} z<0$ and for $p>1$ if $0<\operatorname{Re} z<1$. Interpolation now yields $\left\|T_{0} g\right\|_{p} \leq C\|g\|_{p}, p>1$, for simple $g$.

Given $f \in L^{p}, p>1$, we can find functions of type $\Phi * g$ which converge to $f$ in $L^{p}$ and a.e. A simple limit argument then gives

$$
\left\|\mu_{j(x)} * f(x)\right\|_{p} \leq C\|f\|_{p} .
$$

Letting $N \rightarrow \infty$ and choosing the optimal $j(x)$ for each $N$, we obtain Proposition 1 and, thus, Theorem 1.

3. Some proofs in $\mathbf{R}^{3}$. We first give a simple proof of Theorem 1 for $n=2$ in the case $\alpha_{1}+\alpha_{2}=1$. Write the integral in (1.1) as a sum of integrals over those subsets where $2^{-m} \leq x_{2} / x_{1}<2^{-m+1}$. In these integrals, we change variables 
through $t=x_{1}, \lambda=2^{m} x_{2} / x_{1}$. Hence,

$$
\begin{aligned}
& M f(y) \leq \sup _{h_{1}, h_{2}>0} \int_{1}^{2} d \lambda \sum_{m \in \mathbf{Z}} \frac{2^{-m}}{h_{1} h_{2}} \\
& \cdot \int_{0}^{\min \left(h_{1}, 2^{m} h_{2} / \lambda\right)} t\left|f\left(y_{1}-t, y_{2}-2^{-m} \lambda t, y_{3}-2^{-\alpha_{2} m} \lambda^{\alpha_{2}} t\right)\right| d t \\
& \leq \sup _{h_{1}, h_{2}>0} \int_{1}^{2} d \lambda \sum_{m \in \mathbf{Z}} \frac{2^{-m}}{h_{1} h_{2}} \min \left(h_{1}, \frac{2^{m} h_{2}}{\lambda}\right)^{2} \\
& \text {. } \sup _{h>0} \frac{1}{h} \int_{0}^{h}\left|f\left(y_{1}-t, y_{2}-2^{-m} \lambda t, y_{3}-2^{-\alpha_{2} m} \lambda^{\alpha_{2}} t\right)\right| d t \\
& \leq \sup _{h_{1}, h_{2}} \int_{1}^{2} \sum_{m \in \mathbf{Z}} \min \left(2^{-m} \frac{h_{1}}{h_{2}}, 2^{m} \frac{h_{2}}{h_{1}} \lambda^{-2}\right) M_{\mathrm{lac}}^{\lambda} f(y) d \lambda \\
& \leq C \int_{1}^{2} M_{\mathrm{lac}}^{\lambda} f(y) d \lambda
\end{aligned}
$$

where $M_{\text {lac }}^{\lambda}$ denotes the maximal function along the lacunar directions $\left(1,2^{-m} \lambda\right.$, $\left.2^{-\alpha_{2} m} \lambda^{\alpha_{2}}\right), m \in \mathbf{Z}$. It is shown in [3] that $M_{\text {lac }}^{\lambda}$ is bounded on $L^{p}, p>1$, and the bound is uniform in $\lambda$, as seen by linear mappings. An integration in $\lambda$ now ends the proof.

ProOF OF TheOREM 2. Consider first the case $b=0$. The two parabolic maximal function operators

$$
M_{a}^{1} f(x)=\sup _{h>0} \frac{1}{h} \int_{0}^{h}\left|f\left(x_{1}-s, x_{2}, x_{3}-a s^{2}\right)\right| d s
$$

and

$$
M_{c}^{2} f(x)=\sup _{h>0} \frac{1}{h} \int_{0}^{h}\left|f\left(x_{1}, x_{2}-s, x_{3}-c s^{2}\right)\right| d s
$$

are bounded on $L^{p}, p>1$; see [8]. Their composition dominates our operator; in fact, $M f \leq M_{a}^{1} M_{c}^{2} f$, and the conclusion follows in this case.

Assume now that $b \neq 0$. It is enough to consider $h_{1}, h_{2}$, with $h_{1}<h_{2}$ in (1.1).

When $c \neq 0$, we write $F=a^{\prime}\left(x_{1}^{\prime}\right)^{2}+c\left(x_{2}^{\prime}\right)^{2}$, where $x_{1}^{\prime}=x_{1}$ and $x_{2}^{\prime}=x_{2}+$ $b x_{1} / 2 c$. The domain of integration in (1.1) is contained in $R^{\prime}=\left\{\left(x_{1}^{\prime}, x_{2}^{\prime}\right): 0<x_{1}^{\prime}<\right.$ $\left.h_{1},\left|x_{2}^{\prime}\right|<C h_{2}\right\}$, whose area is $2 C$ times larger. The mean in (1.1) can thus be replaced by that over $R^{\prime}$, and the case $b=0$ applies.

When $c=0$, we write $F=x_{1}^{\prime} x_{2}^{\prime}$ instead where $x_{1}^{\prime}=x_{1}$ and $x_{2}^{\prime}=a x_{1}+b x_{2}$. Now we can apply Theorem 1 to the mean over the same rectangle $R^{\prime}$ as before. This completes the proof of Theorem 2 .

\section{REFERENCES}

1. W. Littman, Fourier transforms of surface-carried measures and differentiability of surface averages, Bull. Amer. Math. Soc. 69 (1963), 766-770.

2. A. Nagel and E. Stein, On certain maximal functions and approach regions, Adv. in Math. 54 (1984), 83-106.

3. A. Nagel, E. Stein and S. Wainger, Differentiation in lacunary directions, Proc. Nat. Acad. Sci. U.S.A. 75 (1978), 1060-1062. 
4. A. Nagel and S. Wainger, $L^{2}$ boundedness of Hilbert transforms along surfaces and convolution operators homogeneous with respect to a multiple parameter group, Amer. J. Math. 99 (1977), 761785.

5. P. Sjögren, Fatou theorems and maximal functions for eigenfunctions of the Laplace-Beltrami operator in a bidisk, J. Reine Angew. Math. 345 (1983), 93-110.

6. ___ Admissible convergence of Poisson integrals in symmetric spaces, Preprint no. 1985-4, Chalmers Univ. of Technology and Univ. of Göteborg, Göteborg, Sweden.

7. E. Stein, Singular integrals and differentiability properties of functions, Princeton Univ. Press, Princeton, N. J., 1970.

8. E. Stein and S. Wainger, Problems in harmonic analysis related to curvature, Bull. Amer. Math. Soc. 84 (1978), 1239-1295.

9. E. Stein and G. Weiss, Introduction to Fourier analysis on Euclidean spaces, Princeton Univ. Press, Princeton, N.J., 1971.

10. R. Strichartz, Singular integrals supported on submanifolds, Studia Math. 74 (1982), 137-151.

11. J. Vance, $L^{p}$-boundedness of the multiple Hilbert transform along a surface, Pacific J. Math. 108 (1983), 221-241.

Department of Mathematics, Chalmers University of TeChNology and UniVERSITY OF GÖTEBORG, S-412 96 GÖTEBORG, SWEDEN (Current address of Hasse Carlsson and Peter Sjögren)

Institute of Mathematical and Physical Sciences, University of Troms $\varnothing$, P.O. BOX 953, N-9001 TROMS $\varnothing$, NORWAY (Current address of J.-O. Strömberg) 